TUMORIGENESIS

\section{Changing signals}

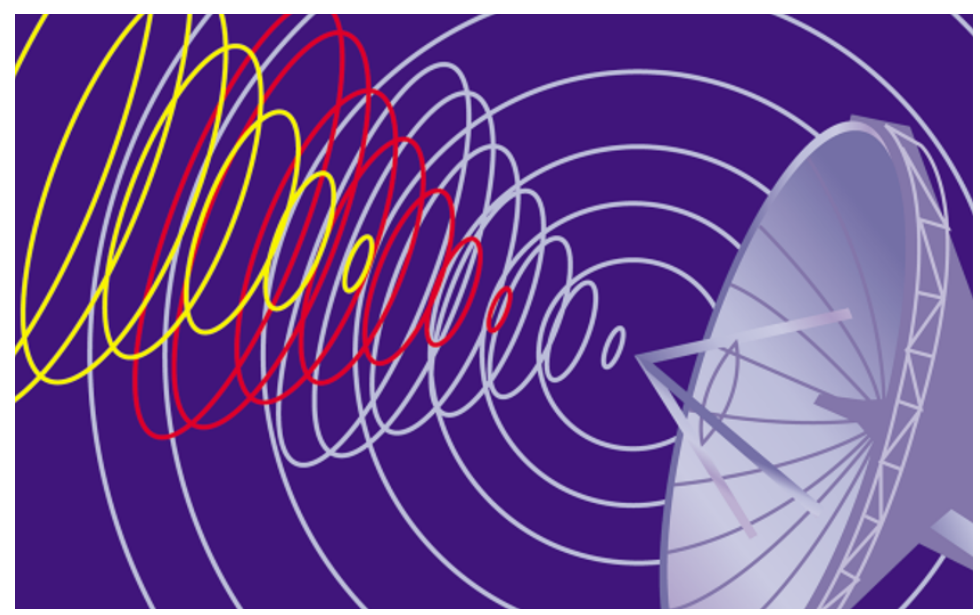

The transforming properties of RAS have been apparent since its discovery 30 years ago. But why does oncogenic RAS cause cell-cycle arrest when introduced into many types of normal cells? Normal cells seem to contain safety mechanisms that inhibit cell-cycle progression when RAS is activated. So, for RAS to have a positive effect on proliferation, additional genetic changes must occur to overcome cell-cycle checkpoint controls and to contribute to the development of tumours. In the April issue of Molecular and Cellular Biology, Alison Lloyd and colleagues show that the p53 and RB pathways have distinct roles in this process.

The authors used primary rat Schwann cells - these proliferate indefinitely, but retain the cell-cycle controls that are normally lost during the immortalization process to investigate which genetic changes cooperate with oncogenic Ras and contribute to a transformed phenotype. They first investigated the contribution of $\mathrm{p} 53$, by expressing oncogenic Ras and either a dominant-negative mutant of p53
(Ras/dnp53) or SV40 large T antigen (Ras/LT), which inhibits the p53 and $\mathrm{Rb}$ pathways. Both Ras/dnp53 and Ras/LT cells were able to proliferate in the absence of mitogens, but only Ras/LT cells had a high proliferative capacity in soft agar and within monolayers. So, perhaps both the RB and $\mathrm{p} 53$ pathways must be inhibited for RAS to overcome anchorage dependence and contact inhibition.

Deletion analysis of LT showed that the amino terminus, which inhibits the $\mathrm{Rb}$ pathway, co-operates with Ras and dnp53 to allow growth in soft agar. Cells expressing dnp53 and activated Ras sometimes spontaneously acquire anchorage independence, with expression of the cyclin-dependent kinase inhibitor Ink4a being lost in 5 out of 10 cases due to promoter methylation. As INK4A lies upstream of the RB family in the tumour-suppressor pathway, its loss would be expected

\title{
Don't forget the handbrake
}

Mirror, signal, manoeuvre....and it's all systems go. You might think that all you need to induce tumour regression are the conditions to stimulate an immune response, but tumour immunologists are discovering that you won't get far unless you remember to release the handbrake.

Tumour cells express antigens that are recognized by the host immune system, but they are generally poorly immunogenic. The most obvious explanation for this is that antigen presentation is inefficient. Various vaccination strategies have been tested to improve antigen presentation, such as engineering autologous tumour cells to secrete granulocyte-macrophage colony-stimulating factor (GM-CSF), which induces dendritic-cell (DC) maturation to produce professional antigen-presenting cells.

In Phase I clinical trials of this strategy, although an antitumour response is elicited in the short term, patients go on to develop progressive disease. Cytotoxic $\mathrm{T}$ lymphocyte antigen 4 (CTLA4) might be one of the brakes that holds the immune system back, and transient antibody- mediated blockade of CTLA4 in combination with vaccination in animal models can increase tumour rejection. However, once the brakes have been removed, it's difficult to stop the action, and mice treated with CTLA4-blocking antibodies develop fatal

lymphoproliferation. So, Allison, Dranoff and colleagues set out to test the safety of this combination therapy in humans.

A single intravenous dose of the CTLA4blocking antibody MDX-CTLA4 was tested in seven patients with metastatic melanoma and two patients with ovarian carcinoma, all of whom had previously been involved in various Phase I vaccination trials.

In the two patients with ovarian carcinoma and three of the patients with melanoma - who had been previously vaccinated with GM-CSF-secreting tumour cells - there was evidence of enhanced tumour necrosis. There was no evidence of tumour destruction in the other four patients with melanoma, who had been vaccinated with melanoma antigen-derived peptides or DCs expressing tumour differentiation antigens. This indicates that the effectiveness of CTLA4 blockade depends on the specific nature of the immune response and that CTLA4 might not be involved in inhibiting all antitumour responses.

The patients developed low titres of autoantibodies, but there was no clinical evidence of autoimmune disease. One patient developed an acute hypersensitivity reaction to the antibody, but this could be easily controlled with antihistamines. The patients with melanoma developed a skin rash, but not vitiligo (depigmentation), which shows that although tolerance to melanocyte differentiation antigens was affected, the response was mild and melanocytes were not destroyed.

This study is the first to show the clinical safety and efficacy of vaccination and CTLA4 blockade, even when the two are separated temporally. This approach could be extended to other types of cancer, and CTLA4 blockade might also synergize with other cancer treatments that stimulate the immune system, such as radiation and chemotherapy.

KirstyMinton

(2) References and links

ORIGINAL RESEARCH PAPER Hodi, F. S. et al. Biologic activity of cytotoxic T Iymphocyte-associated antigen 4 activity of cytotoxic Tlymphocyte-associated antigen 4
antibody blockade in previously vaccinated metastatic melanoma and ovarian carcinoma patients. Proc. Natt Acad. Sci. USA 100, 4712-4717 (2003) 
to substitute for the loss of RB-family members and provide the ability to grow independently of anchorage. This was confirmed by showing that antisense Ink4a and expression of a Cdk4 mutant that mimics the loss of Ink4a confer anchorage independence to Ras/dpn53 cells.

So, a number of genetic events are needed to change the RAS signal from stop to go. p53 loss confers mitogen independence, but the RB pathway must also be inhibited for cells to overcome contact inhibition and anchorage dependence. This RB-pathway inhibition frequently occurs through loss of INK4A, which provides an explanation for how this common tumour suppressor contributes to the tumour phenotype.

Emma Croager

(2) References and links ORIGINAL RESEARCH PAPER Mitchell, $P$. J. Perez-Nadales, E., Malcom, D. S. \& Lloyd, A. C. Dissecting the contribution of $p 16^{\text {INKAA }}$ and the Rb family to the Ras transformed phenotype. Mol. Cell. Biol. 23, 2530-2542 (2003)

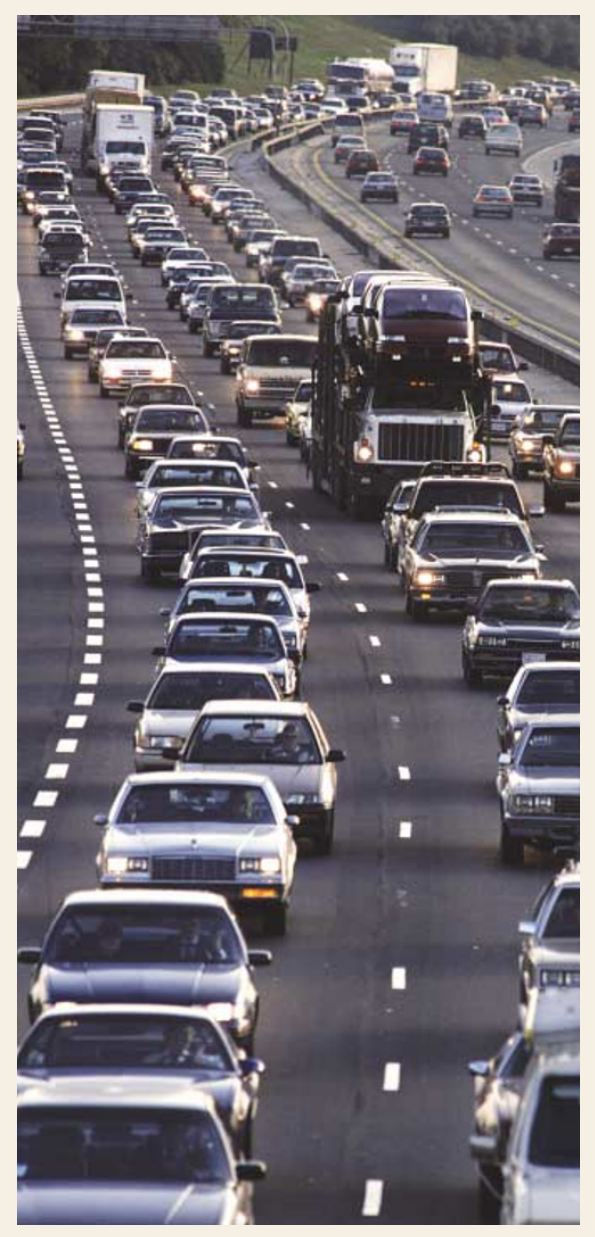

ANGIOGENESIS

\section{Culprits uncovered}

The ability of transformed cells to initiate angiogenesis is a crucial step in tumour progression. Twenty years ago, it was shown that $M Y C$ and RAS oncogenes cooperate to transform cells. Now, Robert Weinberg and colleagues show that cooperation between these two genes is also required for developing neovasculature.

Reporting in the March issue of Cancer Cell, they describe the elucidation of the signalling pathway that leads from RAS to MYC, and culminates in the repression of the key anti-angiogenic factor, thrombospondin-1 (TSP1) (see figure).

The authors established embryonic kidney and mammary epithelial human cell lines transformed with SV40 early-region proteins, the catalytic subunit of human telomerase and either low or high levels of oncogenic RAS expression. The cell lines with low RAS expression were unable to develop tumours of more than $1-2 \mathrm{~mm}$ in diameter in nude mice, and could not initiate neoangiogenesis, whereas the cell lines with high RAS expression produced tumours of $1.5 \mathrm{~cm}$ in diameter with established neovasculature.

So, were key regulators of angiogenesis altered in these cell lines? Although overexpression of pro-angiogenic vascular endothelial growth factor (VEGF) did make cells with low RAS expression more tumorigenic, cells with high RAS expression did not produce significantly more VEGF than those with low RAS expression. This indicates that differences in VEGF expression can not explain the marked differences in tumorigenic potential. By contrast, TSP1 levels were greatly reduced in high-RAS-expressing cells compared with lowRAS-expressing cells. Reducing the expression of TSP1 in low-RAS-expressing cells, using TSP1 antisense, enabled the cells to form large vascularized tumours in nude mice. In addition, when high-RAS-expressing cells were transduced with a TSP1 expression construct to increase levels of the factor, tumours were reduced in size and viable cells were limited to a thin layer on the outside of tumour masses.

As RAS signalling stabilizes MYC, and MYC has been implicated in the repression of TSP1, the authors next investigated the role of MYC in these cell lines. When high-RAS-expressing cells were transduced with dominant-negative MYC, TSP1 levels increased, and when intact MYC was introduced into low-RAS-expressing cells, activation of MYC led to a decrease in TSP1 expression. Interestingly, the levels of MYC did not vary among the RAS-expressing cell lines, but examination of phosphorylation status revealed that phosphorylation of two specific residues, one

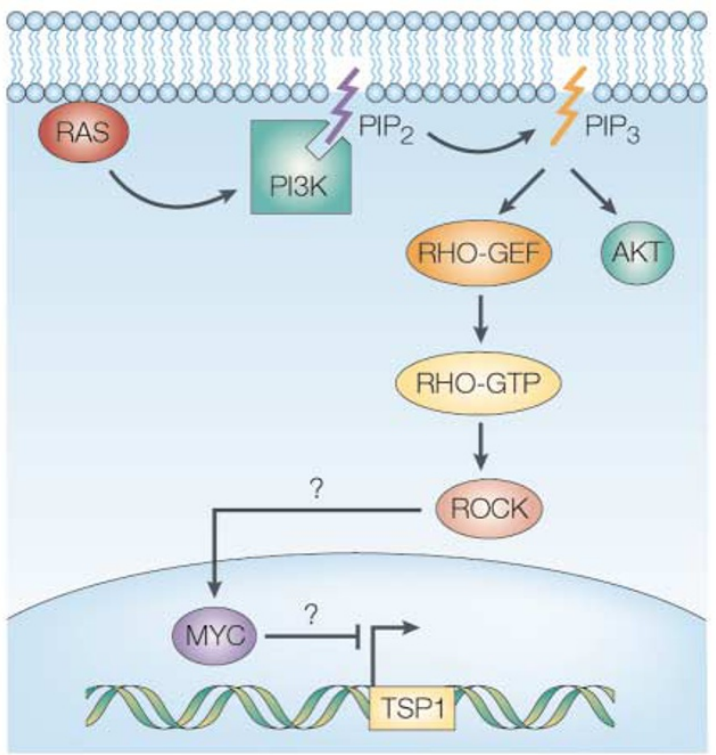

of which is in the transactivation domain, was necessary for MYC's repression of TSP1.

RAS activates three effector pathways, but which one is responsible for suppression of TSP1 expression via MYC? A series of experiments using chemical inhibitors, as well as constitutively active and dominant-mutant constructs of components of pathways downstream of RAS, uncovered the signalling cascade. Weinberg and colleagues showed that signalling occurs via the phosphatidylinositol 3-kinase (PI3K) pathway. The best-studied effect of PI3K is its action on AKT kinase, but constitutively active AKT had no effect on TSP1 levels. By contrast, expression of RHO-GTP - a member of the family of GTPases activated by the PI3K pathway (via guanine exchange factors (GEFs) ) - was higher in highRAS-expressing cells, and a dominant-negative mutant of RHOA relieved the repression of TSP1 in the same cells. The main effectors of RHO are the ROCK protein kinases, and inhibition of these also increased TSP1 levels in high-RAS-expressing cells and prevented phosphorylation of MYC. The mechanism by which MYC represses TSP1 transcription is still unclear.

Having resolved the signalling pathway from RAS to TSP1, the authors validated their findings in three human breast cancer cell lines. TSP1 expression was undetectable in these cells, but TSP1 expression was stimulated using inhibitors of PI3K or ROCK. Further investigation of the relative roles of the anti-angiogenic factor TSP1 and the pro-angiogenic factor VEGF in regulating neoangiogenesis is awaited with great interest.

Ezzie Hutchinson

(2) References and links

ORIGINAL RESEARCH PAPER Watnick, R. S. et al. Ras modulates Myc activity to repress thrombospondin-1 expression and increase tumor angiogenesis. Cancer Cell 3, 219-231 (2003)

WEB SITE

Robert A. Weinberg's lab: http://web.wi.mit.edu/weinberg/pub/ 